\title{
TRAQUELECTOMÍA: UNA ALTERNATIVA TERAPÉUTICA PARA CONSERVAR LA FERTILDAD EN EL CÁNCER CÉRVICO-UTERINO INICIALMENTE INVASOR
}

\author{
Raúl Puente P.', Sergio Guzmán C. ${ }^{1}$, Eduardo Israel A. ${ }^{1}$, Daniel Carpio P. \\ ${ }^{1}$ Sección de Ginecología Oncológica, Instituto de Obstetricia y Ginecología. ${ }^{2}$ Instituto de Histología y Patología, Hospital \\ Clínico Regional de Valdivia, Universidad Austral de Chile
}

\section{RESUMEN}

El mejor conocimiento existente de la diseminación, de la técnica quirúrgica y del manejo perioperatorio, es posible actualmente, realizar cirugía conservadora en las etapas iniciales del cáncer cérvico-uterino, manteniendo incluso la fertilidad. Una de estas técnicas es la traquelectomía radical que siendo un procedimiento localmente radical permite conservar el útero con esos fines. Presentamos aquí un estudio piloto de 3 casos de traquelectomía radical en las que se logró el primer embarazo y parto, sin recurrencias hasta la fecha en ninguna paciente. Para efectuar una traquelectomía radical debe seguirse un estricto proceso de selección, realizándose sólo en centros terciarios de referencia.

\section{PALABRAS CLAVE: Traquelectomía radical, cáncer cérvico-uterino, traquelectomía y embarazo}

\section{SUMMARY}

A reduction in morbidity and preservation of fertility are in some cases a major concern in the treatment of gynecologic cancer. Radical trachelectomy is a local radical surgery which permits to preserve the corpus uteri in situ in selected cases of early invasive cervical cancer. Three patients in which a radical trachelectomy for cervical cancer is performed are presented. A successfully term pregnancy was obtained in one of them. All the patients are free of disease in follow up. Radical trachelectomy must be performed in a tertiary referral centre for gynecological oncology.

KEY WORDS: Radical trachelectomy, cervical carcinoma, trachelectomy and pregnancy

\section{INTRODUCCIÓN}

Según publicaciones recientes, en los últimos años ha habido un aumento del $77 \%$ en la incidencia del cáncer cérvico-uterino invasor en mujeres jóvenes entre 25 y 34 años, los que en su mayoría corresponderían a casos reconocidos dentro de los planes de detección precoz de esta lesión (1). De acuerdo a datos disponibles, un $45 \%$ de los carcinomas etapa IB tratados quirúr- gicamente se presentarían en mujeres bajo los 40 años (2). En consecuencia, sería esperable que un gran número de ellos se diagnosticaran en etapas iniciales, y por lo tanto deberían presentarse como carcinomas de pequeño volumen.

La FIGO ha recomendado la conización cervical como tratamiento para la etapa I A1 en mujeres jóvenes con deseos de mantener la fertilidad, lo que es ampliamente aceptado y se realiza prácticamente en todo el mundo (3). No obstante, para 
los carcinomas cérvico-uterino etapas I A 2, I B1 y II A el problema es diferente ya que por tratarse de lesiones mayores al I A1 se han aplicado y se continúan aplicando en general las 2 modalidades de tratamiento tradicional existentes, a saber, la cirugía radical tipo II o III con linfadenectomía pelviana, o el uso de radioterapia pélvica con o sin braquiterapia. Está reconocido que el uso de cualquiera de estas 2 modalidades de tratamiento para las etapas descritas asegurará excelentes resultados en tasas de sobrevida, pero su aplicación también significará la pérdida completa de la fertilidad.

Gracias a que en la actualidad disponemos de un mejor conocimiento de la diseminación del cáncer cérvico-uterino, asistimos a una tendencia creciente hacia la realización de cirugías limitadas o moduladas en las etapas I, o II iniciales, las que nos podrían permitir por un lado, disminuir la morbilidad postoperatoria de la cirugía radical sin comprometer el éxito del tratamiento, y por otro, intentar preservar la fertilidad sin el peligro de subtratamiento.

Pertenece al ginecólogo francés Daniel Dargent el mérito de llevar adelante el tratamiento del carcinoma cérvico-uterino etapa I B1 o II A inicial, mediante la denominada traquelectomía radical, que no es sino una modificación de la histerectomía radical efectuada por vía vaginal (operación de Schauta). Esta consiste, en síntesis, en la extirpación total del cuello uterino junto con la mitad de los parametrios, conservando in situ el cuerpo uterino y los anexos (4). Para esto se ha considerado que si en una lesión etapa I A2, o I B1- II A está indicado y es posible realizar una cirugía radical del tipo II por vía abdominal, esta misma cirugía podría efectuarse por vía vaginal a una lesión confinada a la porción externa del cuello uterino que tuviera la misma etapificación. La resección de todo el cuello uterino más la mitad de los parametrios se constituiría indudablemente en un tratamiento suficiente, si en el estudio histológico de la pieza se reconocieran márgenes terapéuticos, en especial hacia el endocérvix.

Sabemos que el riesgo de tener compromiso distal de los parametrios es muy bajo si los ganglios pelvianos son negativos $(5,6)$, por lo que la ejecución de la linfadenectomía pelviana como primer paso de la intervención, con estudio de los ganglios por biopsia contemporánea, aportaría los datos necesarios sobre diseminación de la enfermedad. De esta manera se lograría la mantención del cuerpo uterino y por ende, la capacidad reproductiva de la paciente $(4,7)$.
Consideramos que con los antecedentes mencionados, más la existencia de una significativa proporción de mujeres que desearían mantener la fertilidad, la traquelectomía radical más la linfadenectomía pelviana es hoy un recurso terapéutico válido para las etapas I A2, I B1 y || A inicial del cáncer de cuello uterino, pudiendo acceder a esta técnica siempre que se cumplan las condiciones requeridas para su correcta aplicación.

Presentamos aquí la experiencia de nuestras 3 primeras pacientes sometidas a traquelectomía radical más linfadenectomía pelviana luego de producirse el primer embarazo y parto exitoso después de la operación.

\section{MATERIAL Y MÉTODO}

Las 3 pacientes sometidas a traquelectomía radical tenían un cáncer cérvico uterino etapa I B1 con histología de carcinoma epidermoide (2 pacientes) y de adenocarcinoma (1 paciente), con una edad promedio de 26,3 años (rango: 22-31 años). Todas fueron evaluadas por el equipo de la Sección de Ginecología Oncológica del Hospital Clínico Regional de Valdivia, centro de referencia terciario para el manejo de patologías neoplásicas del aparato genital femenino.

En la Tabla I se presentan los criterios para seleccionar a las pacientes que se incorporaban al protocolo $(1,8)$.

La etapificación clínica de las pacientes se cumplió siguiendo los criterios preconizados por la FIGO, incluyendo además ecotomografía de la pelvis para evaluar el volumen cervical y la cercanía al istmo o la infiltración del mismo. Este procedimiento, aunque discutible, ha demostrado tener bastante fiabilidad en nuestro medio, y por ello se ha continuado aplicando. Ninguna paciente tuvo TAC ni MRI previa.

\section{Tabla I}

\section{CRITERIOS DE ELEGIBILIDAD PARA REALIZAR LA TRAQUELECTOMÍA RADICAL}

- Intenso deseo de embarazarse o fuerte motivación psicológica para conservar el útero

- Tumor s a $2 \mathrm{~cm}$ con escasa o nula extensión al endocérvix (por biopsias múltiples, cono frío, o LEEP)

- Ganglios pelvianos negativos a la biopsia rápida

- Sin compromiso de espacios linfovasculares

- Seguridad de control y seguimiento

- Ser realizado en un centro terciario de referencia

- Consentimiento informado 
Todas recibieron detallada información sobre el procedimiento y sus eventuales desventajas frente al tratamiento quirúrgico convencional existente para su neoplasia, así como también de la eventualidad de abortar el procedimiento cambiándolo por una cirugía radical en caso de la aparición de ganglios positivos a la biopsia rápida, o el hallazgo intraoperatorio de un tumor de insospechadas dimensiones. Se les informó además de la posibilidad de tener que realizar una nueva cirugía o adicionar un nuevo tratamiento al ya realizado, de acuerdo al resultado histopatológico de los ganglios, los parametrios y el cuello uterino, incluidos sus factores pronósticos.

En el preoperatorio las pacientes recibieron profilaxis anticoagulante con heparina de bajo peso molecular además de antibióticos de amplio espectro. Antes de proceder a la traquelectomía radical, todas fueron sometidas a linfadenectomía pelviana bilateral completa por vía extraperitoneal empleando la laparotomía de Maylard, sin abrir el peritoneo. Se extirparon los ganglios ilíacos externos, ilíacos internos, ilíacos primitivos desde el cruce ureteral hacia distal, y los obturadores superficiales. Todos los ganglios obtenidos fueron enviados a biopsia rápida procediéndose a efectuar la traquelectomía radical siempre y cuando el

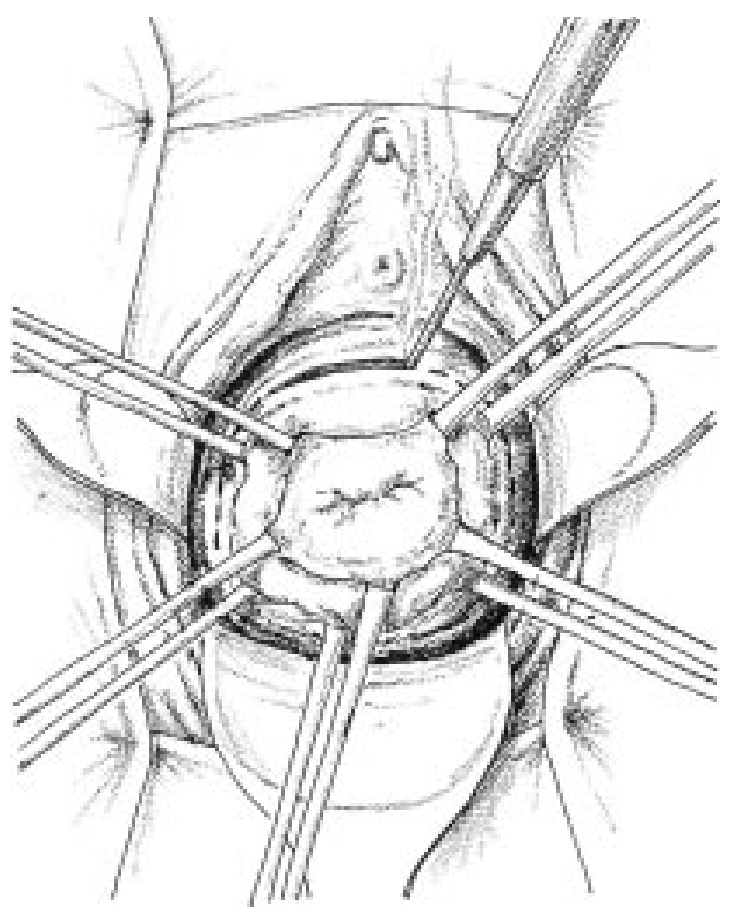

Figura 1. Incindiendo circunferencialmente la vagina. informe de los ganglios fuera negativo. Para la traquelectomía radical se siguió la técnica de Dargent (4), con algunas de las modificaciones sugeridas por Shepherd (1).

Técnica quirúrgica. Las pacientes se colocaron en posición de litotomía, como para realizar una histerectomía vaginal. A ninguna se le practicó incisión de Schuchard. Se infiltró con suero fisiológico un circulo vaginal completo que comprendió todo su calibre, distal unos $2 \mathrm{~cm}$ del cuello, efectuándose luego una incisión circunferencial en ese mismo lugar de la mucosa vaginal, disecándola para separarla de los planos profundos y poder conformar un cilindro vaginal que pudiera cubrir y proteger el cuello como un capuchón, de la misma manera que para una operación de Schauta (Figura 1). Se desarrolló entonces el espacio vesicovaginal y los espacios paravesicales, quedando el parametrio anterior al medio de ambos (Figura 2). En éste se identificó el uréter por palpación, procediéndose a movilizarlo y separarlo hacia arriba de manera de poder despejar la rama cervical de la arteria uterina para seccionarla y ligarla sin contratiempos (Figura 3). Los parametrios laterales se identificaron entre los espacios paravesical por delante y pararectal por atrás seccionándolos en la mitad de su recorrido hacia la pelvis. Se cuidó en forma muy especial que el uréter ni la arteria uterina se incluyeran en el pinzamiento de los cardinales (Figura 4). Seguidamente se proce-

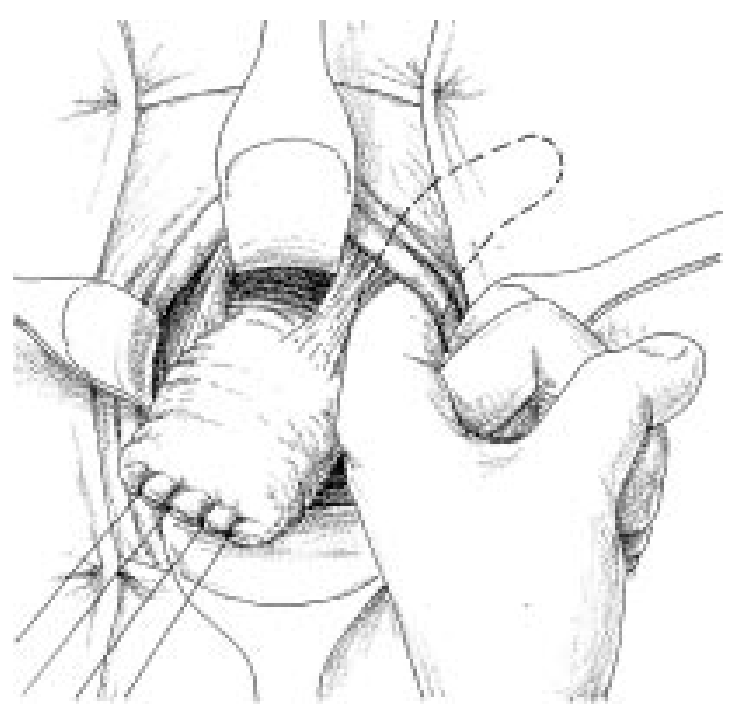

Figura 2. Se ha cubierto el cuello con la mucosa vaginal. Se desarrolla el espacio paravesical apreciándose el espacio vésico-vaginal y el parametrio anterior. 


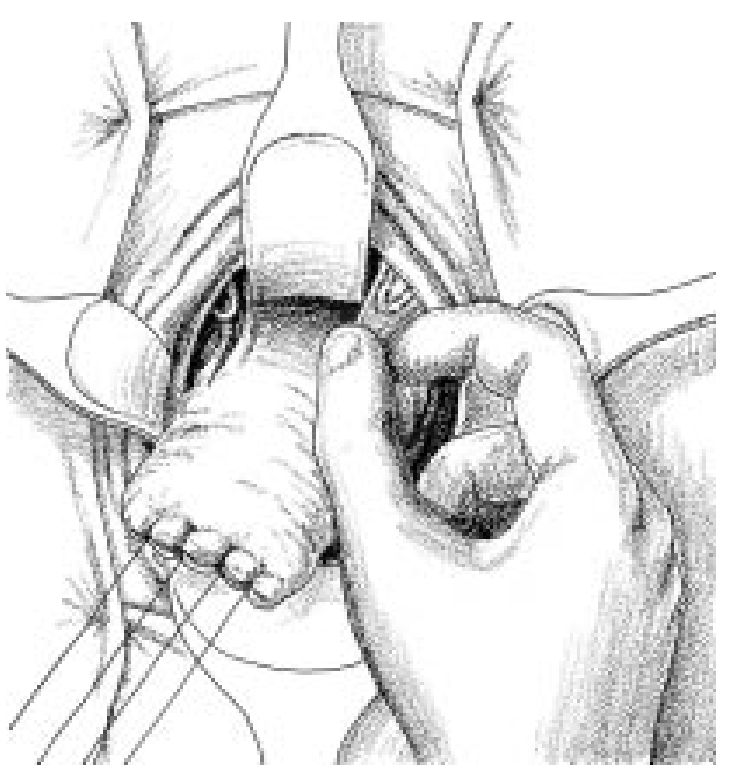

Figura 3. Palpación del uréter en el parametrio anterior.

dió a pinzar y seccionar los vasos uterinos (Figura 5). Finalmente se desarrolló el espacio recto vaginal, despejando los ligamentos útero-sacros sin abrir el fondo de saco de Douglas, seccionándolos a $2 \mathrm{~cm}$ del cuello.

El conducto endocervical se identificó usando un dilatador de Hegar № 6, seccionando el cuello a nivel del istmo con electrobisturí (Figura 6). El cuello en conjunto con los parametrios fue enviado a biopsia rápida para reconocer y asegurar márgenes resectivos terapéuticos, insistiéndose en reconocer la distancia entre el tumor y el margen endocervical proximal. A continuación se realizó un cerclaje con prolene $1 / 0$, anudándolo sobre el dilatador de Hegar (Figura 7). Posteriormente se utilizó suturas de ácido poliglicólico para unir el epitelio vaginal sobre el istmo, cuidando de mantener el conducto endocervical siempre permeable (Figura 8).

A todas se les recomendó usar anticoncepción hormonal durante los primeros 6 meses.

\section{RESULTADOS}

La primera paciente fue operada en diciembre de 1999, y la última en abril de 2002, por lo que el promedio de seguimiento es 33 meses (rango: 2451 meses). Las características clínicas de las pacientes así como las de diagnóstico de su neoplasia se describen en la Tabla II.

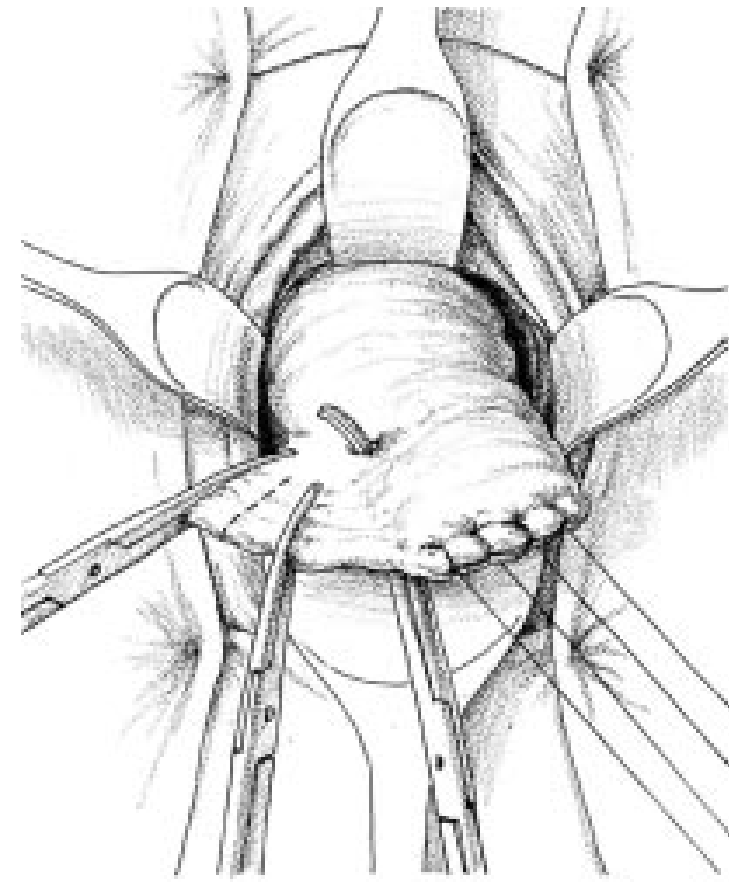

Figura 4. Identificación y sección del parametrio lateral (línea punteada).

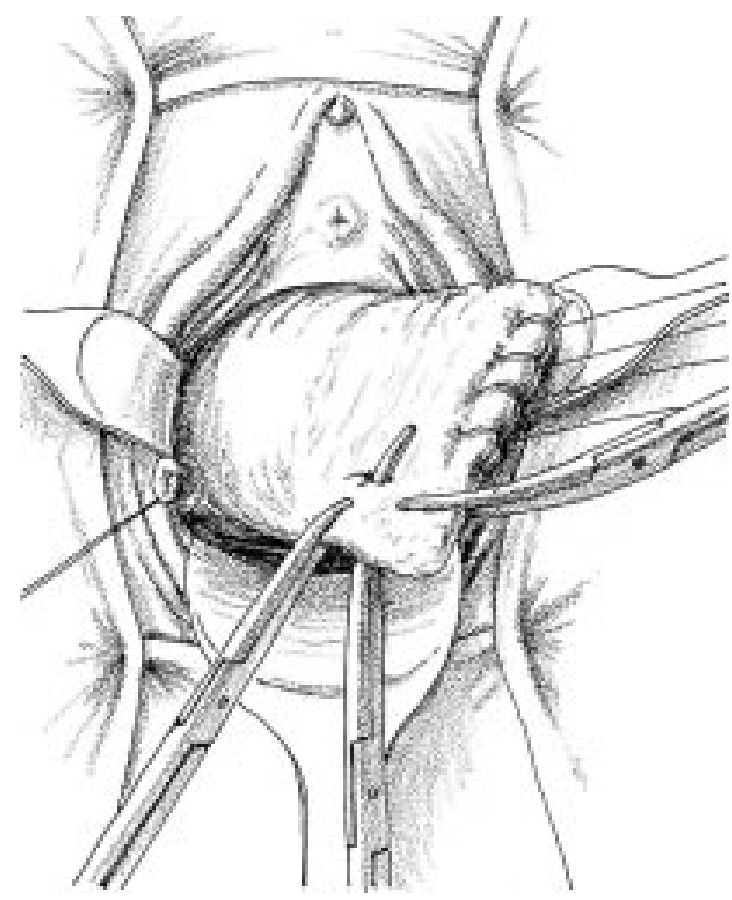

Figura 5. Identificación y pinzamiento de los vasos uterinos. 


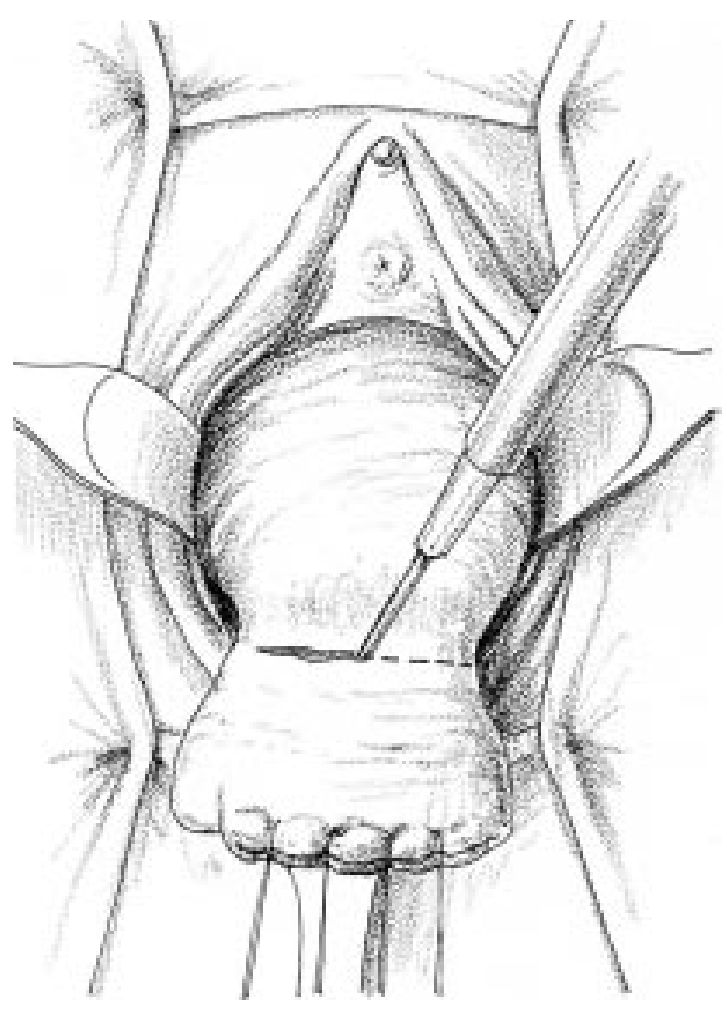

Figura 6. Sección del cuello con electrobisturí a nivel ístmico.

Se obtuvo un promedio de 23 ganglios por paciente (rango: 19-25). La altura promedio de los cuellos obtenidos fue de $31,6 \mathrm{~mm}$ y el promedio del mango vaginal de $24 \mathrm{~mm}$. Los parametrios a su vez midieron $23,3 \mathrm{~mm}$ en promedio. La pieza operatoria, sus características y el seguimiento se muestran en la Tabla III.

De las 3 pacientes operadas, la primera estaba siendo estudiada por infertilidad secundaria antes de operarse y después de su intervención decidió postergar todo estudio adicional tendiente a corregirla. Actualmente no usa anticoncepción pero no desea embarazo.

La segunda paciente (nuligesta) por razones de seguridad decidió usar anticoncepción oral más allá de los 6 meses recomendados, totalizando 16 meses de uso. Actualmente se encuentra en el sexto mes con intención de embarazo, no lográndolo aún.

La tercera paciente, multípara de 2 hijos vivos, manifestó su intención de embarazarse apenas transcurridos los 6 meses de seguridad recomendados desde su operación, embarazándose al quinto mes. La evolución de su embarazo fue

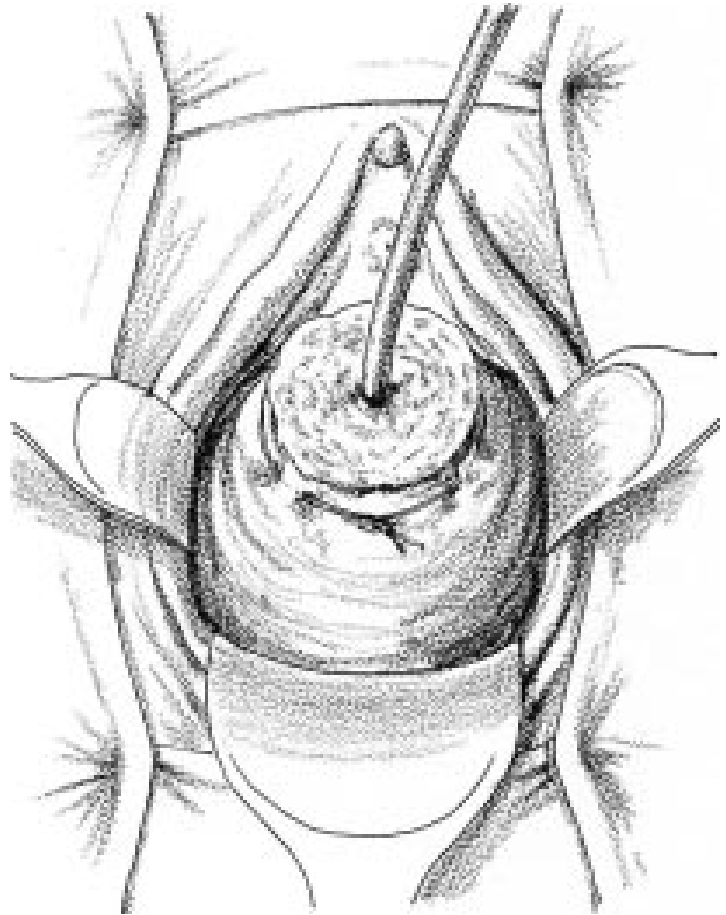

Figura 7. Cerclaje del cérvix usando dilatador de Hegar $\mathrm{N}^{\circ} 6$ en el endocérvix.

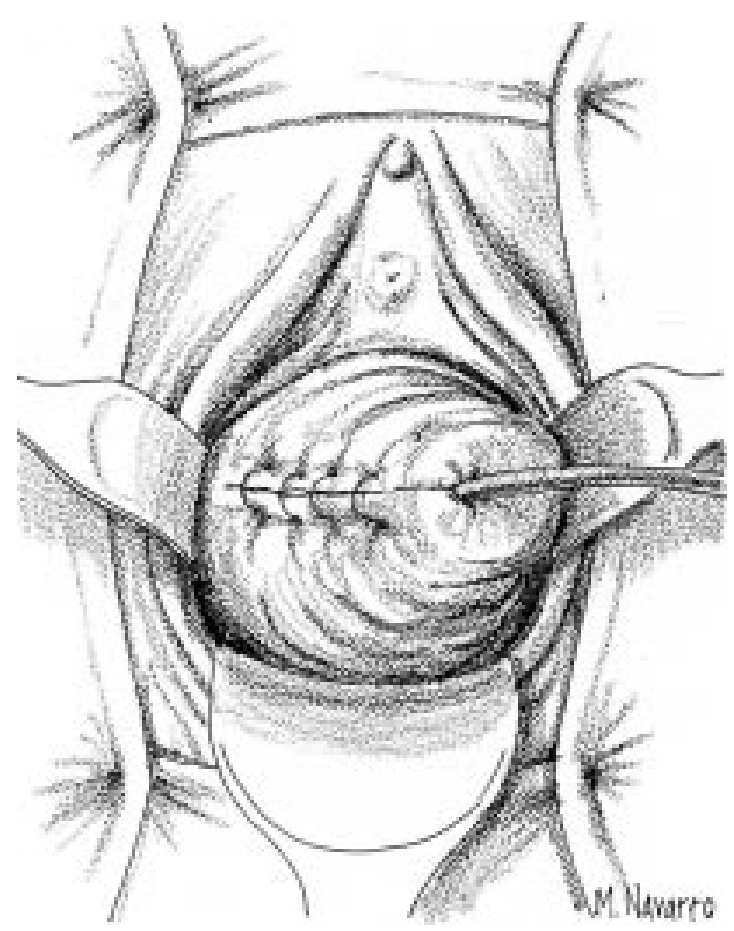

Figura 8. Cierre de la vagina sobre el istmo con puntos de ácido poliglicólico. 
Tabla II

CARACTERÍSTICAS CLÍNICAS Y DIAGNÓSTICO PREOPERATORIO

\begin{tabular}{lcccll}
\hline Caso & Edad & Paridad & Etapa & Histología & Diagnóstico/tamaño TU \\
\hline 1 & 31 años & 10001 & IB1 & Escamoso & $\begin{array}{l}\text { Cono LEEP incompleto / } 8 \mathrm{~mm} \text { lateral } \\
4 \mathrm{~mm} \text { profundidad }\end{array}$ \\
2 & 22 años & 00000 & IB1 & Adenocarcinoma & Bp. múltiple/15x20 mm, invasor, macroscópico \\
3 & 26 años & 20102 & IB1 & Escamoso & Cono LEEP/27x25 mm, x 3 mm profundidad \\
\hline
\end{tabular}

Tabla III

CARACTERÍSTICAS ANATOMO-PATOLÓGICAS DEFINITIVAS Y SEGUIMIENTO

\begin{tabular}{llllrr}
\hline Caso & $\begin{array}{l}\text { Longitud } \\
\text { del cuello }\end{array}$ & Manguito vaginal & $\begin{array}{l}\text { Resultado de la biopsia } \\
\text { definitiva }\end{array}$ & $\begin{array}{c}\text { Seguimiento } \\
\text { (meses) }\end{array}$ & $\begin{array}{c}\text { Status } \\
\text { actual }\end{array}$ \\
\hline 1 & $30 \mathrm{~mm}$ & $20 \mathrm{~mm}$ mínimo & NIE III c/brotes microscópicos & 51 & SEE $^{*}$ \\
2 & $35 \mathrm{~mm}$ & $35 \mathrm{~mm}$ mínimo & $\begin{array}{l}\text { TU de } 15 \times 8 \mathrm{~mm} \text { c/profundidad } \\
\text { invasión } 8 \mathrm{~mm}\end{array}$ & 25 & SEE* $^{*}$ \\
3 & $30 \mathrm{~mm}$ & $17 \mathrm{~mm}$ mínimo & Sin lesión residual & 24 & SEE $^{*}$ \\
\hline
\end{tabular}

*Sin evidencia de enfermedad.

normal con 4 controles de su condición cervical, con citologías negativas y colposcopias normales. Tuvo su parto por cesárea a las 38 semanas en el Hospital de Puerto Montt el 7 de diciembre de 2003 sin complicaciones, se obtuvo un recién nacido vivo y sano de 2.580 gramos, situación que motivó la publicación de esta serie.

Las 3 pacientes han efectuado sus controles sistemáticamente, y todas se encuentran al día sin evidencia de enfermedad, con citologías y colposcopias negativas.

\section{DISCUSIÓN}

La experiencia en traquelectomía radical en todo el mundo es aún limitada, siendo las series más numerosas las publicadas por Dargent, y los canadienses Covens y Roy (2). Series menores han sido reportadas por Shepherd (1) en Gran Bretaña, autores escandinavos (9) y de otros pocos países, pero tenemos la impresión que en Sudamérica la técnica aún no está incorporada y en Chile parece ser la primera publicación sobre el tema.

Sin duda cada día existe mayor aceptación para efectuar cirugías menos radicales y que se modulen de acuerdo al volumen tumoral y a las posibilidades de diseminación que éste presente. Estudios de Burghardt y Pickel (10) y de Winter
(11) además de nuestra propia experiencia, han demostrado que cuando los tumores son pequeños, y con mayor razón, cuando los ganglios pelvianos son negativos, la posibilidad de tener compromiso distal de los parametrios es muy baja $(5,6)$. Desde el punto de vista terapéutico convencional, para éstos casos bastaría con efectuar una histerectomía radical tipo II, condición en la que la sección de los parametrios se realiza en la mitad del trayecto hacia la pelvis. Siendo así, parece lógico inferir que si hacia la parte distal del parametrio la diseminación es exigua, algo semejante sucedería hacia la parte alta del endocérvix, en especial si la lesión primaria asienta principalmente en el exocérvix. Burghardt y Holzer (12) establecieron hace ya varios años que desde el punto de vista terapéutico no se necesita extirpar el fondo uterino ni los anexos en el manejo de tumores de pequeño volumen. En consecuencia, la traquelectomía radical representaría la aplicación del concepto de cirugía menos radical indicada para tumores pequeños, cuya aceptación se da hoy en día en numerosas situaciones.

Aunque muchos especialistas tengan la idea de que esta cirugía no parece un tratamiento suficiente para un carcinoma de cuello uterino etapa I B1 o II A, para que la aplicación de ella sea exitosa es preciso tener en cuenta no sólo la edad de la paciente y su deseo de mantener la fertili- 
dad, sino también un adecuado estudio preoperatorio que permita descartar la presencia de un gran tumor cervical o que éste comprometa las cercanías del istmo uterino. Para ello hemos utilizado con bastante éxito el diagnóstico ecográfico, complementado con histeroscopia. Está claro que si en el intraoperatorio las condiciones nos mostraran lesiones tumorales mayores o diseminadas fuera del cuello, el procedimiento debe ser abortado.

Para realizar la traquelectomía radical consideramos indispensable efectuar en primer lugar la linfadenectomía pelviana por vía extraperitoneal, con biopsia rápida de los ganglios obtenidos, que no deberían ser menos de 10 por lado para representar adecuadamente el estado ganglionar pelviano y poder decidir así una adecuada cirugía radical limitada (13). En nuestros casos obtuvimos un promedio de 23 ganglios, cifra que se compara favorablemente con lo que ha sido publicado en otras series.

La linfadenectomía extraperitoneal tiene la enorme ventaja de evitar las adherencias postoperatorias al útero o a los anexos que podrían comprometer la fertilidad, e indudablemente se podría realizar por laparoscopia (14), aunque nosotros hemos preferido la incisión de Maylard por si algún ganglio pelviano resultara positivo a la biopsia rápida. En ese caso se suspendería la ejecución de la traquelectomía radical programada, sustituyéndola por una cirugía radical tipo III-IV con linfadenectomía sistemática de todo la pelvis más linfadenectomía aórtica hasta la mesentérica inferior, o por radioterapia. En los 3 casos presentados, no hubo ganglios positivos ni en la biopsia rápida ni en el informe definitivo.

El estudio histopatológico de las piezas quirúrgicas de nuestras pacientes demuestra que las traquelectomías radicales han sido técnicamente óptimas y por lo tanto son comparables con las otras experiencias reportadas. Sin duda alguna se ha extirpado la mitad de los parametrios, que en promedio superan los $23 \mathrm{~mm}$, en tanto Dargent informa un promedio de $10 \mathrm{~mm}$ para los mismos.

Aunque se ha considerado que las lesiones escamosas serían las más susceptibles de ser tratadas mediante traquelectomía radical, es necesario puntualizar que los adenocarcinomas deberían ser tratados en forma similar a los carcinomas escamosos (15) puesto que extirpando todo el cuello desaparecerían los peligros de multifocalidad propios de las lesiones con esa histología. De hecho en nuestra serie se incluye un caso con histología de adenocarcinoma. La posibilidad de tener metástasis ovárica en pacientes con este tipo de carcinoma debe descartarse mediante ultrasonido previo a la cirugía.

Con relación a los embarazos, una de las series reportada por Dargent (4) muestra que en pacientes sometidas a traquelectomía radical se obtuvo un $80 \%$ de embarazos, lográndose parto con hijo vivo en $62,5 \%$ de los mismos. Otros autores han reportado $60 \%$ de embarazos, logrando partos con recién nacido vivo en $50 \%$ de ellos (1). Sobre este punto es necesario entender que aunque la fertilidad pudiera verse afectada por la ejecución misma de la traquelectomía, ésta se realiza en el contexto de pacientes con diagnóstico de carcinoma invasor del cuello uterino, debiendo entenderse que mientras se conserve el útero, siempre existirá la posibilidad de un embarazo.

Desde el punto de vista oncológico la traquelectomía radical al igual que otro procedimiento terapéutico en cáncer obliga a un seguimiento estricto que permita reconocer precozmente persistencias o recidivas de tal modo de manejarlas rápidamente si así sucediera. Las mayores series existentes muestran que las recidivas tendrían una frecuencia que oscilaría entre 10\% (1) y algo más del $14 \%$ (4).

Resulta llamativo que habiendo practicado la primera intervención en 1999, sólo se han operado 3 pacientes hasta la fecha. Para nuestra sorpresa hemos verificado que muchas pacientes que han reunido los requisitos necesarios, han rechazado la proposición de una traquelectomía radical por no parecerles una intervención del todo segura para tratar su cáncer en forma efectiva. La mayoría se ha inclinado por una intervención que -dentro de las posibilidades- les ofrezca las mayores probabilidades de quedar sanas de una vez, sugiriéndonos ser lo más erradicativos posible.

Aunque la traquelectomía radical aparece en gíneco-oncología como una intervención de bajo riesgo, debe ser realizada en un centro de referencia terciario, por especialistas con amplios conocimientos de la cirugía radical de la pelvis, siendo uno de sus puntos críticos el adecuado manejo del uréter, que en esta operación puede sufrir un daño inadvertido si no es movilizado correctamente fuera del campo sujeto a resección. Tampoco puede ofertarse como un tratamiento que reemplace el estándar quirúrgico hoy imperante. Deben seguirse rigurosamente sus indicaciones y condiciones, hasta que los resultados en grandes grupos de mujeres puedan demostrar que se podría extender con seguridad a un mayor de número de pacientes. 
En conclusión, la traquelectomía radical aparece conceptualmente como un intento intermedio de proporcionar un tratamiento locoregional adecuado en mujeres seleccionadas, portadoras de tumores cervicales de pequeño volumen que así pueden conservar el útero y su capacidad reproductiva, y que de otra manera deberían ser consideradas para histerectomía radical por vía abdominal o vaginal. La obtención de un embarazo con hijo vivo de término que reportamos en nuestra experiencia demuestra que con ella se cumplen a cabalidad los objetivos perseguidos.

\section{BIBLIOGRAFÍA}

1. Shepherd JH, Crawford RAF, Oram DH. Radical trachelectomy: a way to preserve fertility in the treatment of early cervical cancer. $\mathrm{Br} \mathrm{J}$ Obstet Gynaecol 1998; 105: 912-916.

2. Covens A. Preserving fertility in early cervical $\mathrm{Ca}$ with radical trachelectomy. Contemporary Obstet Gynecol 2003; 2: 46-66.

3. Ngan HYS, Benedet JL, Jones III HW, Bender HG, Pecorelli S. Cancer of the cervix, En Benedet JL, Pecorelli S (Eds). Staging classifications and clinical practice guidelines of gynecologic cancers by the FIGO Committee on Gynecologic Oncology. Elsevier, 2000; 27-40.

4. Dargent D, Roy M. Vaginal radical trachelectomy In: Benedetti-Panici P, Scambia G, Maneschi F, Sevin BU, Mancuso S (eds). Wertheim's Radical Hysterectomy. Roma: Societa Editrice Universo, 1996; 61-69.

5. Puente R, Guzmán S, Israel E, Poblete MT. Does the pelvic lymph nodes predict the parametrial status in cervical cancer stage I B-IIA? Eur J Gynecol Oncol 2001; 22(Suppl 1): 34 (Abstract).
6. Girardi F, Lichteneger W, Tamussino K, Haas J. The importance of parametrial lymph nodes in the treatment of cervical cancer. Gynecol Oncol 1989; 34: 206-11.

7. Dargent D. Laparoscopic assisted vaginal radical hysterectomy; evolution of a concept. CME J Gynecol Oncol 2001, 6: 102-09.

8. Benedetti-Panici P, Cutillo G, Angioli R. Modulation of surgery in early cervical cancer. Critical Reviews in Oncology/ Hematology 2003; 48: 263-270.

9. Goderstad JM, Baekelandt M, van de Putte G, Kristensen G. Radical trachelectomy. The Norwegian experience. Int J Gynecol Cancer 2003; 13: 98 (Abstract).

10. Burghardt E, Pickel H. Local spread and lymph node involvement in cervical cancer. Obstet Gynecol 1978; 52: 138-45.

11. Winter R. Stage IB-IIA-IIB cervical cancer: which kind of radical hysterectomy? In: Benedetti-Panici $P$, Scambia G, Maneschi F, Sevin BU, Mancuso S. (Eds). Wertheim's Radical Hysterectomy. Roma: Societa Editrice Universo, 1996; 15-18.

12. Burghardt E, Holzer E. Diagnosis and treatment of microinvasive carcinoma of the cervix uteri. Obstet Gynecol 1977; 49: 641-653.

13. Puente R, Guzmán S, Israel E, Poblete M T, Del Pozo M. Concordance and validation study of pelvic lymph nodes by frozen section and its use to tailor the radical surgery in patients with cervical carcinoma stage IB-IIA. Int J Gynecol Cancer 2003; 13(Suppl (1): 92 (Abstract).

14. Dargent D. Using radical trachelectomy to preserve fertility in early invasive cervical cancer. Contemporary Ob/Gyn 2000; 5: 23-49.

15. Berek J, Hacker N. Cervical cancer. In: Practical Gynecologic Oncology. $3^{\text {rd }}$ ed. Philadelphia: Lippincott, Williams \& Wilkins 2000: 345-405. 